\title{
The reliability of the submaximal version of the Yo-Yo intermittent recovery test in elite youth soccer
}

\author{
Charlie Owen, Paul Jones, Paul Comfort
}

Objectives: To determine the test-retest reliability of the submaximal version of the Yo-Yo Intermittent Recovery Test - Level 1 (Yo-Yo IR1-sub), which is a valid aerobic assessment.

Design: Test-re-test.

Methods: Elite youth soccer players $(\mathrm{n}=10$, age: $18.8 \pm 0.5$ years, height: $181.1 \pm 4.9 \mathrm{~cm}$, body mass: $74.2 \pm 7.6 \mathrm{Kg})$ completed the Yo-Yo IR1-sub on two consecutive weeks to determine the reliability of a non-exhaustive alternative to maximal aerobic testing. Relative and absolute reliability of the Yo-Yo IR1-sub was assessed for heart rate response and recovery metrics (HR6, HR30, HR60, HR90, HR120, HRR30, HRR60, HRR90 and HRR120).

Results: The Yo-Yo IR1-sub was determined to be a reliable alternative to maximal testing, with the final heart rate at the end of the 6 minute test (HR6) shown to be the most reliable metric $(\mathrm{ICC}=0.96$; $\mathrm{SDD}=5.4 \mathrm{bpm})$. The percentage of heart rate recovered after $2 \mathrm{~min}$ (HRR120) was the most reliable recovery metric (ICC $=0.93$; SDD $=4.6 \%$ ).

Conclusion: If maximal testing is not a feasible option, particularly during the competitive season or during the rehabilitation of an injured player, then a submaximal test is a reliable alternative to monitor fitness in elite young soccer players, with HR6 and HRR120 being the most stable parameters.

(Journal of Trainology 2017;6:31-34)

Key words: non-exhaustive $\boldsymbol{\square}$ heart rate $\boldsymbol{\square}$ recovery $\boldsymbol{\square}$ test-retest $\boldsymbol{\square}$ aerobic performance

\section{INTRODUCTION}

Soccer is an intermittent, team based sport, where repeated high intensity actions are required, interspersed with periods of lower intensity or submaximal work. ${ }^{1}$ The ability to repeat these high intensity actions, such as sprinting and changing direction, across a 90 minute game is primarily governed by a player's aerobic capacity. ${ }^{2}$ Consequently, it is the aerobic performance of soccer players that can determine whether they operate at the elite, sub-elite or non-elite level. ${ }^{3}$ With this in mind, it seems pertinent to have the tools to accurately assess aerobic capacity within a team based sport such as soccer.

The gold standard approach to assess aerobic capacity is via an incremental treadmill protocol using breath by breath gas analysis, ${ }^{4}$ with a maximum oxygen uptake $\left(\mathrm{VO}_{2} \max \right)$ of 60 ml.kg. min $^{-1}$ reported as the benchmark for an elite soccer player. ${ }^{5}$ Higher levels of $\mathrm{VO}_{2} \max$ have been shown to result in increased match performance, including distance covered, number of sprint and number of involvements with the ball. 5,6 However, the assessment of $\mathrm{VO}_{2}$ max via direct gas analysis on a treadmill can be a time consuming method, whilst lacking soccer specific movement patterns, due to the intermittent multi-directional nature of the sport. Several field based soccer specific endurance tests have been developed to better represent the physiological profile of a soccer match, such as the Yo-Yo Intermittent Endurance Test - Level 1 and Level 2 (YoYo IE1, Yo-Yo IE2) ${ }^{4}$ and the Yo-Yo Intermittent Recovery
Test - Level 1 and Level 2 (Yo-Yo IR1, Yo-Yo IR2). ${ }^{7}$ Field assessments ensure that multiple players in a squad can be assessed simultaneously, with minimal equipment required. ${ }^{8}$

The Yo-Yo IR1 and Yo-Yo IE2 tests have starting speeds of $10 \mathrm{~km} \cdot \mathrm{h}^{-1}$ and $11.5 \mathrm{~km} \cdot \mathrm{h}^{-1}$ respectively, with a view of shortening total assessment time, when compared to the slower starting speed $\left(8 \mathrm{~km} \cdot \mathrm{h}^{-1}\right)$ of the Yo-Yo IE1. The difference in starting speeds between tests result in a broad range of distances covered and total work time, ranging from $2564 \pm 554 \mathrm{~m}$ during the Yo-Yo IE2, ${ }^{9}$ therefore it is difficult to directly compare results due to the methodology applied. Following each $2 \times$ $20 \mathrm{~m}$ shuttle, these tests have a rest period of $10 \mathrm{~s}$ and $5 \mathrm{~s}$ respectively. As a result, the Yo-Yo IR1 provides more time to recover and is better regarded as an aerobic, soccer specific test. ${ }^{10}$ The Yo-Yo IR1 requires a maximal effort until volitional exhaustion in order for a true reflection of aerobic capacity to be reported. Distances of $2128 \pm 236 \mathrm{~m},{ }^{11}$ and $2434 \pm$ $248 \mathrm{~m}^{12}$ in youth soccer players and $2455 \pm 488 \mathrm{~m}^{13}$ in senior players, reflect the physically demanding nature of the test, along with a diverse spread of data between players.

Although the testing and monitoring of aerobic performance is important at the elite level of competition, a maximal test of endurance may not always be appropriate for soccer players in season, particularly considering the continued efforts to identify a shorter test. ${ }^{9}$ With the recent literature surrounding training load and injury risk in elite soccer, ${ }^{14}$ a typical Yo-Yo IR1

Received March 6, 2017; accepted May 22, 2017

From the Manchester United Football Club, AON Training Complex, Carrington, Manchester, UK (C.O.) and Directorate of Sport, Exercise and Physiotherapy, University of Salford, Salford, Greater Manchester, UK (C.O., P.J., P.C.)

Communicated by Takashi Abe, PhD

Correspondence to Dr. Paul Comfort, Directorate of Sport, Exercise and Physiotherapy, University of Salford, Salford, Frederick Road, Salford, Greater Manchester, M6 6PU. UK. Email: P.Comfort@salford.ac.uk

Journal of Trainology 2017;6:31-34 @2012 The Active Aging Research Center http://trainology.org/ 
benchmark of $2300 \mathrm{~m}^{15}$ is a considerable distance, supplementary to the training load of a normal training week. As opposed to administering the Yo-Yo IR1, a more efficient, submaximal version has been utilised; the Yo-Yo Intermittent Recovery Test - submaximal (Yo-Yo IR1-sub). Non-exhaustive testing is a useful monitoring tool, particularly in-season when physical demands are high or frequent testing is required. ${ }^{9}$ In addition, with the test lasting a total of $6 \mathrm{~min}$, with a total of $720 \mathrm{~m}$ covered, between player comparisons can be made for the same absolute workload.

The Yo-Yo IR1-sub appears to be a valid and useful method of monitoring aerobic fitness over the course of the playing season. ${ }^{16-18}$ The key metric identified during the Yo-Yo IR1sub is the 6 min heart rate at cessation of the test (HR6). ${ }^{16}$ It has been established that HR6 is inversely correlated with Yo-Yo IR1 performance, where $\mathrm{r}=-0.54,{ }^{16} \mathrm{r}=-0.81^{17}$ and $r=-0.81,{ }^{15}$ although identified with elite senior players ${ }^{16,17}$ and sub-elite youth players ${ }^{15}$ respectively. The heart rate response at $6 \mathrm{~min}$ is shown to be a valid measure of capacity ${ }^{19}$ and a reproducible measure of aerobic fitness $(\mathrm{ICC}=0.78)$, albeit in semi-professional players. ${ }^{15}$ However, data regarding the reliability of HR6 is still limited with regard to elite youth soccer players. In addition, the reliability of heart rate recovery indices following the Yo-Yo IR1-sub is yet to be established. Although the reliability of heart rate recovery metrics following the maximal version of the Yo-Yo IR1 at $60 \mathrm{~s}$ $(\mathrm{ICC}=0.72-0.92)$ and $120 \mathrm{~s}(\mathrm{ICC}=0.69-0.85)$ have been reported, ${ }^{20}$ this is yet to be established for the submaximal alternative.

The aim of this study was to establish the reliability for both HR6 and the HR recovery values at 30, 60, 90 and $120 \mathrm{~s}$ following completion of the Yo-Yo IR1-sub. It was hypothesized that HR6 and HRR would be reliable metrics in elite youth soccer and therefore provide an efficient alternative to maximal testing to assess the aerobic fitness of young elite soccer players.

\section{METHODS}

\section{Participants}

Ten elite youth soccer players (age $17.8 \pm 0.5$ years, height $181.1 \pm 4.9 \mathrm{~cm}$, mass $74.2 \pm 7.6 \mathrm{Kg}$ ) were recruited for the current study, with a prior power calculations revealing that this would yield a statistical power $\sim 0.7$ at an a prior alpha level of $p \leq 0.05$. All participants were members of an English Premier League Academy and subject to a full-time training schedule in line with the Premier League's Category 1 programme guidelines. All testing procedures were conducted as part of the player's normal training schedule. The study and any data pertaining to the study were approved by the University Institutional Review Board along with approval from the soccer academy. Participants provided written informed consent or parental assent where appropriate. The participants recruited for the study were all part of the full time U21 squad (U21).

\section{Experimental Approach}

A test-retest design was used to investigate the reliability of the Yo-Yo IR1-sub in elite youth soccer players. Participants performed the Yo-Yo IR1-sub on two occasions, at the same time of day, separated by one week, in order to determine testretest reliability. The training regime on the days prior to testing was standardised, as was dietary intake on the day of testing.

\section{Procedures}

All participants were subject to the Yo-Yo IR1-sub a minimum of six times per season as part of their normal training schedule and were therefore familiar with the test. Participants completed the Yo-Yo IR1-sub on two occasions separated by one week in order to determine test-retest reliability. Due to the players being part of an elite training programme, this allowed preparation between testing sessions to remain consistent.

The Yo-Yo IR1-sub was administered on a 3G artificial surface with all players wearing the appropriate footwear, heart rate belt and global positioning system (GPS) unit. The Yo-Yo IR1-sub consisted of two $20 \mathrm{~m}$ shuttle runs that were incremental in speed, dictated by an audio signal from a compact disc player. ${ }^{7}$ Each shuttle run was separated by a 10 s recovery period in a $5 \mathrm{~m}$ walk zone. Players completed each shuttle in time with the audio signal. Heart rate (HR) was monitored every second via the Polar T31-Coded HR system (Polar Electro, Kempele, Finland) and a $10 \mathrm{~Hz}$ GPS monitoring system (Minimax S4, Catapult Innovations, Canberra, Australia). These systems have been shown to collect valid and reliable data. $^{21}$ The Yo-Yo IR1-sub was terminated after 6 min at $14.5 \mathrm{~km} \cdot \mathrm{hr}^{-1}$ with a total distance of $720 \mathrm{~m}$ completed. Heart rate at this time point was recorded (HR6). Following the test, players were asked to stand still for 2 min whilst HR (bpm) was recorded in $30 \mathrm{~s}$ splits, at 30,60, 90 and $120 \mathrm{~s}$. The absolute HR was converted into a percentage of HR recovered based on the maximum heart rate (MHR) reached during the test $([\mathrm{MHR}-\mathrm{HR} / \mathrm{MHR}] \times 100){ }^{22}$

\section{Statistical Analyses}

Relative reliability of the Yo-Yo IR1-sub was assessed using intraclass correlation coefficients (ICC) for HR6 and heart rate recovery values, from which $95 \%$ confidence intervals (CI) were calculated. Additionally, paired t-tests were performed to compare HR metrics of the two testing sessions. ICC values were interpreted in line with Coppieters et al: ${ }^{23}$ poor $<0.40$, fair $0.4-0.7$, good $0.7-0.9$ and excellent $>0.90$. Absolute reliability was calculated as per Munro et al. ${ }^{24,25}$ in the form of coefficient of variance (CV), standard error of the mean (SEM), with smallest detectable difference (SDD) also calculated $(\mathrm{SDD}=1.96 \times \sqrt{2} \times \mathrm{SEM})$.

\section{RESULTS}

Reliability of the Yo-Yo IR1-sub was good to excellent for seven out of the nine parameters measured (Table 1). ICCs ranged from $0.81-0.96$ with the exception of HR recovery (\%) at 30 and $60 \mathrm{~s}$ (ICC $=0.58$ and 0.68 respectively). The most reliable metric was HR6 (Table 1) where a significant correlation between the two tests was found $(p<0.05)$. There 
Table 1 Descriptive and reliability statistics for the Yo-Yo IR1-sub

\begin{tabular}{lcccccccc}
\hline & $\begin{array}{c}\text { Test 1 } \\
\text { Mean } \pm \text { SD }\end{array}$ & $\begin{array}{c}\text { Test 2 } \\
\text { Mean } \pm \text { SD }\end{array}$ & ICC & $\mathbf{9 5 \%}$ CI & $\begin{array}{c}\text { CV } \\
(\%)\end{array}$ & SEM & $\begin{array}{c}\text { SDD } \\
\text { (absolute) }\end{array}$ & $\begin{array}{c}\text { SDD } \\
(\%)\end{array}$ \\
\hline HR6 (bpm) & $160 \pm 14$ & $161 \pm 14$ & 0.96 & $0.85-0.99$ & 1.6 & 1.9 & 5.4 & 3.4 \\
HR30 (bpm) & $139 \pm 13$ & $137 \pm 17$ & 0.89 & $0.55-0.98$ & 3.9 & 3.5 & 9.7 & 7.0 \\
\hline HR60 (bpm) & $112 \pm 11$ & $115 \pm 17$ & 0.81 & $0.18-0.96$ & 5.6 & 4.4 & 12.3 & 10.8 \\
\hline HR90 (bpm) & $104 \pm 16$ & $106 \pm 16$ & 0.94 & $0.76-0.99$ & 4.2 & 2.7 & 7.5 & 7.2 \\
\hline HR120 (bpm) & $99 \pm 14$ & $99 \pm 15$ & 0.93 & $0.67-0.98$ & 4.4 & 2.7 & 7.5 & 7.6 \\
\hline HRR30 (\%) & $13 \pm 4$ & $15 \pm 4$ & 0.58 & $0.51-0.90$ & 19.5 & 1.9 & 4.3 & 38.4 \\
HRR60 (\%) & $29 \pm 6$ & $28 \pm 7$ & 0.68 & $0.56-0.93$ & 12.2 & 2.5 & 7.0 & 24.3 \\
\hline HRR90 (\%) & $35 \pm 8$ & $34 \pm 7$ & 0.90 & $0.54-0.98$ & 5.9 & 1.7 & 4.6 & 13.3 \\
\hline HRR120 (\%) & $38 \pm 9$ & $38 \pm 9$ & 0.93 & $0.69-0.98$ & 5.7 & 1.7 & 4.6 & 12.2
\end{tabular}

SD = Standard Deviation; ICC = Intraclass Correlation Coefficient; CI = Confidence Interval; CV = Coefficient of Variation; SEM = Standard Error of the Mean; SDD = Smallest Detectable Difference

was no significant difference between test 1 and test 2 for all nine variables measured $(p>0.05)$.

\section{DISCUSSION}

Heart rate at submaximal intensities is sensitive to changes in maximal performance, and therefore such non-exhaustive testing may be a useful and reliable fitness monitoring tool ${ }^{9}$, especially in season. The findings of the current study identified that the Yo-Yo IR1-sub is reliable assessment method in young elite soccer players, with absolute heart rate at cessation of the 6 minute test (HR6) highlighted as the most reliable metric $(\mathrm{ICC}=0.96)$ (Table 1$)$. Importantly small changes in HR6 ( $\geq 6 \mathrm{bpm},>3.4 \%$ ) highlight a meaningful change. This observation suggests that the acute heart rate response to submaximal exercise is the most reproducible measure to monitor aerobic fitness.

The absolute heart rate recovery metrics $(\mathrm{bpm})$ were reliable, with HR90 (ICC $=0.94)$ and HR120 $(\mathrm{ICC}=0.93)$ providing the most reliable information, with changes $>7.2 \%$ and $>7.6 \%$ respectively, considered meaningful changes in response to training or fatigue (Table 1). The absolute HRR metrics were all more reliable than their relative counterparts, yet the relative recovery metrics still provided fair to excellent data. It must be noted that HRR90 and HRR120 were both excellent with regard to reliability ( $\mathrm{ICC}=0.90$ and 0.93 respectively), but it was HRR30 and HRR60 that were somewhat less reliable ( $\mathrm{ICC}=0.58$ and 0.68 respectively), with changes of $>7.0 \%$ and $10.8 \%$ considered meaningful. This may be due to the greater number of beats recovered in the first 30 and $60 \mathrm{~s}$ when compared to 90 and $120 \mathrm{~s}$ (22 and 24 vs. 9 and 6 beats respectively). However, this is the first study to provide reliability data for heart rate recovery at 30,60, 90 and $120 \mathrm{~s}$ in both absolute recovery and percentage recovery formats. The SEM for all nine heart rate metrics for the Yo-Yo IR1-sub was lower than the SDD (Table 1), therefore it is likely that the Yo-Yo IR1-sub can be used to detect small changes in aerobic fitness for young elite soccer players.

Previous research also identified HR6 as the most reliable measure of aerobic fitness $(\mathrm{CV}=2.2 \%){ }^{15}$ The current study reported better relative reliability $(\mathrm{CV}=1.6 \%)$ for HR6 than the study by Fanchini et al. ${ }^{15}$ perhaps due to the elite nature of the participants, who are more familiar with the monitoring protocols administered in an elite programme. The absolute heart rate recovery metrics (bpm) were also comparable to previously reported data. ${ }^{12,20}$ Both absolute $(\mathrm{ICC}=0.81-0.96)$ and relative $(\mathrm{CV}=1.6-5.6 \%)$ reliability values in the current study were in agreement to those calculated in previous studies ${ }^{12,20}$ where the ICC ranged from $0.72-0.93$ and the CV ranged from $1.1-4.1 \%$. However, there is no comparable data for the reliability of the relative heart rate recovery metrics in young elite soccer players, with this study providing novel information on the reliability of such information. It seems that the reliability of the relative heart rate recovery values improve with each 30 s split, with HRR120 proving the most reliable.

It must be noted that although the Yo-Yo IR1-sub provides a submaximal alternative to aerobic testing, the heart rate at cessation of the 6 min test (HR6) reported some inter-individual differences with HR6 values ranging from 145 to $186 \mathrm{bpm}$. With differing levels of aerobic fitness within a squad of players, although the external workload is exactly the same ( 720 $\mathrm{m})$, the internal workload differs between players. As a result, their recovery is not from the same starting point, which may help explain the lower relative reliability values in the first 30 and $60 \mathrm{~s}$. Players with a higher HR6 typically recover a greater number of beats in the first $60 \mathrm{~s}$, compared to those players with a low HR6. Although there are certain benchmarks for the aerobic fitness of an elite young soccer player, it seems they can have a low acute response or a quicker initial recovery to the same absolute workload.

Finally, although maximal testing is an important component of an elite monitoring programme, non-exhaustive testing provides a reliable alternative. Future research may seek to identify the relationship between submaximal and maximal aerobic exercise in young elite soccer players and whether this is indicative of match performance. Secondly, no study to date has identified whether heart rate recovery differs with age following submaximal exercise in young elite soccer players and whether the Yo-Yo IR1-sub is indeed a submaximal test for 
players younger than this study has reported.

\section{CONCLUSION}

If maximal testing is not feasible in-season due to increased training and match demands, a submaximal alternative to monitor the fitness of elite youth soccer players has been shown to be reliable, with changes in HR6 $\geq 6$ bpm ( $>3.4 \%$ ) highlighting a meaningful change. The Yo-Yo IR1-sub could be used to monitor seasonal changes in aerobic fitness when conducted on a regular basis or to identify improvements in aerobic performance when a player is returning from injury. With regard to using HRR as a measure of aerobic fitness, absolute HRR is more reliable than relative HRR, with HR 2 min post exercise providing the most reliable measure for both. Changes $>7.5$ bpm (4.6\%) for HR120 and HRR120 may imply that a meaningful improvement in aerobic fitness has taken place.

\section{FUNDING STATEMENT}

No funding was received in support of this investigation.

\section{CONFLICT OF INTEREST}

The authors have no conflict of interest.

\section{REFERENCES}

1. Reilly T, Bangsbo J, Franks A. Anthropometric and physiological predispositions for elite soccer. J Sports Sci 2000;18:669-683.

2. Stolen T, Chamari K, Castagna C, Wisloff U. Physiology of soccer: an update. Sports Med 2005;35:501-536.

3. Vaeyens R, Malina RM, Janssens M, et al. A multidisciplinary selection model for youth soccer: the Ghent Youth Soccer Project. Br J Sports Med 2006;40:928-934; discussion 934.

4. Metaxas TI, Koutlianos NA, Kouidi EJ, Deligiannis AP. Comparative study of field and laboratory tests for the evaluation of aerobic capacity in soccer players. $J$ Strength Cond Res 2005;19:79-84.

5. Helgerud J, Engen LC, Wisloff U, Hoff J. Aerobic endurance training improves soccer performance. Med Sci Sports Exerc 2001;33:1925-1931.

6. McMillan K, Helgerud J, Macdonald R, Hoff J. Physiological adaptations to soccer specific endurance training in professional youth soccer players. Br J Sports Med 2005;39:273-277.

7. Bangsbo J. The physiology of soccer - with special reference to intense intermittent exercise. Acta Physiol Scand Suppl 1994;619:1-155.

8. Castagna C, Impellizzeri FM, Chamari K, Carlomagno D, Rampinini E. Aerobic fitness and yo-yo continuous and intermittent tests performances in soccer players: a correlation study. J Strength Cond Res 2006;20:320325 .

9. Bradley PS, Mohr M, Bendiksen M, et al. Sub-maximal and maximal Yo-Yo intermittent endurance test level 2: heart rate response, reproducibility and application to elite soccer. Eur J Appl Physiol 2011;111:969-978.

10. Castagna C, Impellizzeri F, Cecchini E, Rampinini E, Alvarez JC. Effects of intermittent-endurance fitness on match performance in young male soccer players. J Strength Cond Res 2009;23:1954-1959.

11. Markovic G, Mikulic P. Discriminative ability of the Yo-Yo intermittent recovery test (level 1) in prospective young soccer players. J Strength Cond Res 2011;25:2931-2934.

12. Deprez D, Fransen J, Boone J, Lenoir M, Philippaerts R, Vaeyens R. Characteristics of high-level youth soccer players: variation by playing position. J Sports Sci 2015;33:243-254.

13. Ferrari Bravo D, Impellizzeri FM, Rampinini E, Castagna C, Bishop D, Wisloff U. Sprint vs. Interval Training in Football. Int J Sports Med 2008;29:668-674.

14. Hulin BT, Gabbett TJ, Lawson DW, Caputi P, Sampson JA. The acute:chronic workload ratio predicts injury: high chronic workload may decrease injury risk in elite rugby league players. $\mathrm{Br} J$ Sports Med 2015;50:231-236.

15. Fanchini M, Schena F, Castagna C, et al. External Responsiveness of the Yo-Yo IR Test Level 1 in High-level Male Soccer Players. Int J Sports Med 2015;36:735-741.

16. Bangsbo J, Iaia FM, Krustrup P. The Yo-Yo intermittent recovery test : a useful tool for evaluation of physical performance in intermittent sports. Sports Med 2008;38:37-51.

17. Krustrup P, Mohr M, Amstrup T, et al. The yo-yo intermittent recovery test: physiological response, reliability, and validity. Med Sci Sports Exerc 2003;35:697-705.

18. Bradley PS, Di Mascio M, Bangsbo J, Krustrup P. The maximal and submaximal versions of the Yo-Yo intermittent endurance test level 2 are simply reproducible, sensitive and valid. Eur J Appl Physiol 2012;112: 1973-1975.

19. Mohr M, Krustrup P. Yo-Yo intermittent recovery test performances within an entire football league during a full season. J Sports Sci 2013;32(4):315327.

20. Deprez D, Coutts AJ, Lenoir M, et al. Reliability and validity of the Yo-Yo intermittent recovery test level 1 in young soccer players. J Sports Sci 2014;32:903-910.

21. Rampinini E, Alberti G, Fiorenza M, et al. Accuracy of GPS devices for measuring high-intensity running in field-based team sports. Int J Sports Med 2014;36:49-53.

22. Lamberts RP, Lemmink KA, Durandt JJ, Lambert MI. Variation in heart rate during submaximal exercise: implications for monitoring training. $J$ Strength Cond Res 2004;18:641-645.

23. Coppieters M, Stappaerts K, Janssens K, Jull G. Reliability of detecting 'onset of pain' and 'submaximal pain' during neural provocation testing of the upper quadrant. Physiother Res Int 2002;7:146-156.

24. Munro AG, Herrington LC. Between-session reliability of four hop tests and the agility T-test. $J$ Strength Cond Res 2011;25:1470-1477.

25. Munro AG, Herrington LC. Between-session reliability of the star excursion balance test. Phys Ther Sport 2011;11:128-132. 